\title{
I Physics: A Field Theory for Gravity
}

The first root of general relativity is the spectacular empirical success of Maxwell electromagnetism, which is the basis of our electric and electronic technology.

Maxwell theory is a field theory. This means that electric and magnetic interactions are not understood as forces acting at a distance between charges (as in Coulomb), but rather as local interactions carried around at a finite speed by a field: the electromagnetic field.

General relativity does the same for gravity. It does not describe gravity as a force between masses that acts at a distance (as in Newton) but as a local interaction carried around at finite speed by a field: the gravitational field.

General relativity is the field theory of the gravitational field, just as Maxwell theory is the field theory of the electromagnetic field. Maxwell theory has been the principal source of inspiration for Einstein in building general relativity.

The need for a field theory for gravity appeared clear to Einstein because of special relativity. I assume the reader is familiar with the basics of special relativity, and in the next section I spell out in detail why special relativity implies that gravity should be described by a field.

\section{I.I SPECIAL RELATIVITY}

\section{- The physical meaning of Galilean relativity}

Newtonian mechanics is invariant under Galilean transformations such as

$$
x^{\prime}=x-v t,
$$


which express the fact that position and velocity are relative physical quantities. That is, the position $x$ and the velocity $v$ of an object are only defined with respect to another object (called, in this context, the reference system).

In equation (1.1), $x$ is the 'position', defined as the distance from a reference object $O$, while $x^{\prime}$ is the distance from a second reference object $O^{\prime}$ moving at a constant speed $V$ with respect to $O$. The quantity $t$ is the time measured by a clock. The invariance of Newtonian mechanics follows from the fact that its fundamental law is

$$
F=m a
$$

and the acceleration $a=d^{2} x(t) / d t^{2}$ does not change under (1.1). Indeed the acceleration with respect to $O^{\prime}$ is $a^{\prime}=d^{2} x^{\prime}(t) / d t^{2}=d^{2} / d t^{2}$ $(x(t)-v t)=d^{2} x(t) / d t^{2}=a$. Hence if $(1.2)$ is true for the position $x$ defined with respect to $O$, it also holds true for the position $x^{\prime}$ defined with respect to $O^{\prime}$.

It follows that it is impossible to distinguish uniform rectilinear motion from stasis using mechanical experiments. Position and velocity are only defined as relative to something else.

This implies that it is impossible to label spacetime events with a preferred spatial position variable $x$.

That is, given two events happening at different times, it is meaningless to say that they happen 'at the same position $x$ ', unless we specify (explicitly or implicitly) a reference object with respect to which position is determined.

'To remain at the same place' with respect to a moving train, with respect to the Earth, with respect to the Sun, or with respect to the Galaxy, have different meanings. A mother telling 'stop moving' to her child on a train does not mean that the child should jump off the train and stop moving with respect to the Earth. 'To remain at the same place' has no sense, unless we specify with respect to what. See the first two panels of Figure 1.1. This is Galilean relativity. 


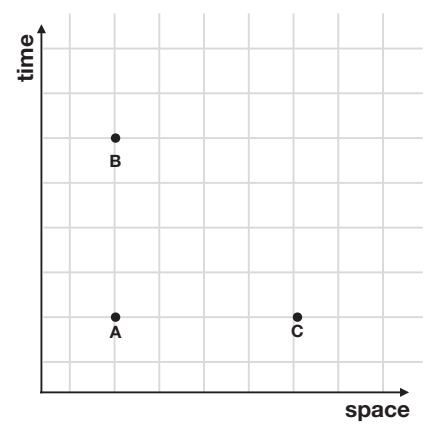

Intuitive spacetime structure Two events (A and $\mathbf{B}$ ) happen at the same point. Two events (A and $\mathbf{C}$ ) happen at the same time.

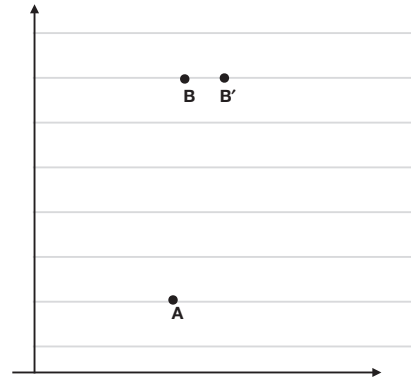

\section{Galilean relativity}

'At the same point' has no absolute meaning.

B happens at the same point as $\mathbf{A}$ with respect to the Earth.

B' happens at the same point as A with respect to the Galaxy.

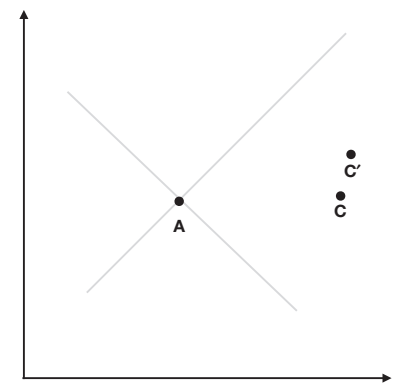

Special relativity

'At the same time' has no absolute meaning.

$\mathbf{C}$ happens at the same time as $\mathbf{A}$ with respect to the Earth

$\mathbf{C}^{\prime}$ happens at the same time as $\mathbf{A}$ with respect to the Galaxy.

FIGURE I.I The structure of spacetime; non-relativistic intuition, Galilean, and special relativity 


\section{- The physical meaning of special relativity}

The Maxwell equations are not invariant under (1.1). Lorentz and Poincare realised that they are instead invariant under a different set of transformations, such as

$$
x^{\prime}=\gamma(x-v t), \quad t^{\prime}=\gamma\left(t-v x / c^{2}\right),
$$

which we call today Lorentz transformations. Here $\gamma=1 / \sqrt{1-v^{2} / c^{2}}$. While the meaning of $x^{\prime}$ was clear to Lorentz and Poincare (it is the distance from a moving object), the meaning of $t^{\prime}$ remained obscure until Einstein.

In 1905, Einstein clarified this meaning by realising that if $t$ is the time measured by a clock moving together with the reference object $O$, then an identical clock moving together with the object $O^{\prime}$ will measure $t^{\prime}$ rather than $t$. That is, identical clocks moving with respect to one another measure different times. This is what Einstein understood in 1905.

This is not a matter of perspective or definitions. It is a physical fact. Consider two identical clocks separated and then brought back together. Say one of the two moves inertially (no acceleration) between the separation and the reunion and measures the time lapse $t$ between separation and reunion. Say the other clock moves at a (possibly variable) speed $v$ with respect to the first. Then, when they meet again, the first will be in advance of the second. If the square of the speed $v$ of the second clock is constant, the second clock measures the time lapse

$$
t^{\prime}=\frac{1}{\gamma} t<t
$$

(If the velocity varies as $v(t)$, then $t^{\prime}=\int_{0}^{t} d \tau \sqrt{1-v^{2}(\tau) / c^{2}}<t$.) The time measured by a clock between two given events depends on the motion of the clock. It is maximal for a clock moving inertially between the two events.

It follows from this property of time intervals that it is impossible to label spacetime events with a unique physically preferred 
time $t$. That is, given two events happening in different locations, it is meaningless to say that two events happen 'at the same time t', unless we specify (explicitly or implicitly) a reference object with respect to which time is determined.

In other words, 'to happen at the same time' with respect to a moving train, with respect to the Earth, with respect to the Sun, and with respect to the Galaxy, have different meanings. See the third panel of Figure 1.1. To ask what is happening 'now' on Andromeda is a question that has no meaning. This is 'special relativity'.

Notice that it is an extension of Galilean relativity from space to time: Galilean relativity is the discovery that 'to be at the same place' at different times is an ill-defined notion, while special relativity is the discovery that 'to happen at the same time' in different places is an equally ill-defined notion.

\section{I.2 FIELDS}

\section{- Special relativity and Coulomb's law}

A consequence of this discovery is that it makes no sense to say that a force acts at a distance instantaneously: when unqualified, 'instantaneously' is meaningless.

This may seem to be in contradiction to Coulomb's law, which states that two charges $e$ and $e^{\prime}$ at a distance $r$ act on each other with a repulsive force

$$
F=\frac{e e^{\prime}}{r^{2}} .
$$

[Before reading ahead, try to answer the following: how can this law be compatible with special relativity?]

If Coulomb's law were a universal law, it would contradict special relativity. But it is not a universal law: it is only valid in the static limit in which the charges do not move or move slowly with respect to one another. In this case, they themselves define the reference system where the law holds. 
To see why Coulomb's law does not have universal validity, consider what happens if we rapidly take away one of the two charges. Does the other one immediately cease to feel the electric force?

The answer is, of course, negative, because information does not travel faster than light: for a time $t=r / c$, the remaining charge keeps feeling the force. During this time, a disturbance of the electromagnetic field travels across the space between the charges at the speed of light, and only when it reaches the second charge does the force on it change. Hence, Coulomb's law is compatible with special relativity only because it is the static, non-relativistic limit of the interactions carried by a field. This is the observation that motivates general relativity.

\section{- Special relativity and Newton's law}

Let us come to gravity. According to Newton's law, two masses $m$ and $m^{\prime}$ at a distance $r$ act on each other with the attractive force

$$
F=G \frac{m m^{\prime}}{r^{2}} .
$$

If this was a universal law, it would contradict special relativity. Indeed: what happens if we rapidly move one of the two masses away from the other? Does the other one instantaneously cease to feel the gravitational force? If special relativity is correct, this cannot be, because information does not travel faster than light: for a time $t=r / c$, the remaining mass keeps feeling the force. During this time, a disturbance of a gravitational field must travel across space at the speed of light, and only when it reaches the second mass will the gravitational force on it change. For this to be possible, there should exist a field describing the degrees of freedom of what travels between one mass and the other.

Therefore, special relativity implies that Newton's law (1.6) is not a universal law: it is a static, non-relativistic limit, valid only when the masses do not move rapidly with respect to each other. Away from this limit, gravity should be described by a field 


\begin{tabular}{lll}
\hline & Electromagnetism & Gravity \\
\hline Static limit & $F=\frac{e e^{\prime}}{r^{2}}$ & $F=G \frac{m m^{\prime}}{r^{2}}$ \\
& Coulomb's law & Newton's law \\
\hline Full theory & Maxwell's field theory & General relativity \\
\hline
\end{tabular}

FIGURE I.2 The logic that led Einstein to general relativity. Coulomb's law is compatible with special relativity only because it is the static limit of a field theory: Maxwell's electrodynamics. Similarly, to be compatible with special relativity Newton's law must be the static limit of a field theory: general relativity.

theory, capable of accounting for the finite speed of propagation of the interaction. General relativity is such a field theory. See Figure 1.2.

\section{- The structure of general relativity}

Maxwell theory is defined by (i) a field: the electromagnetic field, (ii) a force law: the equation that describes how charges move in the field, called the Lorentz force equation, and (iii) field equations: the Maxwell equations.

In parallel, as we shall see, general relativity is defined by (i) a field: the gravitational field, (ii) a law that describes how masses move under the action of this field: the 'geodesic equation', and (iii) field equations: the Einstein equations. See Figure 1.3. This is the structure of the theory that I am going to describe in this book.

However, there is an aspect of gravity that makes it sharply different from electromagnetism. The gravitational field is also related to the geometrical structure of spacetime. Discovering this connection has been Einstein's everlasting contribution. This is discussed in the next chapter. 


\begin{tabular}{lll}
\hline & Electromagnetism & General relativity \\
\hline Field & Maxwell potential & Gravitational field \\
& $A_{a}(x)$ & $g_{a b}(x)$ \\
\hline Particle eq. of $m$. & Lorentz force & Geodesic eq. \\
& $\ddot{X}^{a}=\frac{e}{m} F^{a} b^{b}$ & $\ddot{X}^{b}=-\Gamma^{a} b c^{b} \dot{X}^{c}$ \\
\hline Field equations & Maxwell eqs. & Einstein eqs. \\
& $D_{a} F^{a b}=4 \pi J^{b}$ & $R_{a b}-\frac{1}{2} R g_{a b}+\lambda g_{a b}=8 \pi G T_{a b}$ \\
\hline
\end{tabular}

FIGURE I.3 Comparison of the structure of electromagnetism and general relativity. The quantities in the equations will be defined and discussed in the following sections: the gravitational field $g_{a b}$ in Section 3.2; the Levi-Civita connection $\Gamma_{b c^{\prime}}^{a}$ constructed with first derivatives of $g_{a b}$, in Section 3.2.1; the Ricci tensor $R_{a b}$, constructed with second derivatives of $g_{a b}$, in Section 3.2.3; the cosmological constant $\lambda$ in Section 4.3; and the energy momentum tensor $T_{a b}$ in Chapter 5. 